\title{
RESPIRATORY MICROBIOTA DISORDERS IN CHILDREN WITH NEUROLOGI- CAL DISABILITIES AT REPEATED RESPIRATORY DISEASES
}

\section{O. Koreniuk}

The relevance of the problem of recurrent respiratory diseases in children with severe neurological pathology is due to the high frequency, severity of clinical symptoms and the protracted nature of their exacerbations, as well as the tendency to chronicity of the pathological process.

The aim of the research was to study the respiratory microbiota to optimize antibacterial therapy of recurrent respiratory diseases.

Materials and methods: the 16 children with severe neurological pathology at repeated respiratory diseases were examined. We used a microbiological examination of the upper respiratory tract using a deep smear from the oropharynx to identify a possible causative agent of the disease.

Research results: the pronounced imbalance of the respiratory microbiota, corresponding to II and III degrees of dysbiotic disorders, was revealed in the examined children. The feature of the microflora of the upper respiratory tract was the dominance of Pseudomonas aeroginosa, intestinal opportunistic microflora (Klebsiella pneumoniae, Proteus vulgaris) and their frequent combination with Streptococcus pyogenus, Staphylococcus aureus and yeast-like mushrooms of the Candida genus. The maximum degree of respiratory microbiota disorders was noted in the group of children with congenital malformations of the brain and correlated with the severity of bronchopulmonary diseases. The analysis of the pathogenic microflora sensitivity to antibiotics showed high resistance of the identified microorganisms to most medicines.

Conclusions: respiratory pathology in children with neurological disabilities is characterized by a recurrent and chronic bronchitis with a tendency of a protracted course which determines the duration of antibiotic therapy. The use of a non-invasive technique for the respiratory microbiota studying makes it possible to identify suspected pathogens of bronchopulmonary diseases and optimize their treatment

Keywords: respiratory microbiota, repeated respiratory diseases, disabled children, neurological pathology

Copyright (C) 2021, O. Koreniuk.

This is an open access article under the CC BY license (http://creativecommons.org/licenses/by/4.0).

\section{Introduction}

The problem of recurrent respiratory diseases in children with severe neurological pathology is still relevant. The frequency and severity of the clinical course of acute respiratory diseases in neurological patients is due to the presence of a number of aggravating factors: pseudobulbar syndrome, dysphagia, chronic microaspiration, forced position of the patient, hypotonia of the respiratory muscles, absence or weakness of the cough reflex, decreased immunity, as well as chronic carriage of resistant nosocomial flora.

A feature of respiratory pathology in children with disabilities is a tendency to protracted course, which requires long-term, multi-stage antibiotic therapy. Unfortunately, the empirical method of choosing an antibiotic for the treatment of a respiratory disease is most often used in practice, which leads to the ineffectiveness of the therapy and its prolongation. In this regard, the most important issue is the use of techniques that allow to optimize treatment as a result of the correct selection of a medicine.

According to experimental data of recent years, the fact of the presence of microflora in human lungs has been confirmed and a direct relationship has been noted between the microbiota of the upper and lower respiratory tract $[1,2]$. The study of the physiological role of the upper respiratory tract microbiota is of particular interest when considering the concept of the unity of the airways, according to which the upper and lower respiratory tract is considered a single morphological and functional unit $[2,3]$. The anatomical features of the airway structure allow rapid microbial immigration to the lower airways [2].

One of the most important factors contributing to the penetration of bacterial flora into the lower respiratory tract and determining the state of the lung microbiota is microaspiration [4]. Microbial immigration can occur by inhalation of air with microbial induration in the nasopharynx, microaspiration and direct dispersion along the mucosal surfaces of the upper respiratory tract.

Recent studies have shown that the detection of a pathogen in the culture of material from the upper and lower respiratory tract does not necessarily indicate its role as a causative agent of acute bronchopulmonary disease. An assessment of the severity of dysbiosis is required. The isolation rate of opportunistic microflora in a titer of $10^{6}$ bacterial cells $/ \mathrm{m}^{3}$ and above indicates its participation in the development of the pathological process $[1,5]$.

However, long-term persistence of an infectious agent in patients with acute bronchopulmonary diseases can lead to an imbalance of the immune reactivity and 
the formation of a chronic bronchopulmonary inflammatory process. Increased colonization of the respiratory tract by pathogenic and opportunistic flora in patients with recurrent and chronic respiratory diseases is a significant risk factor contributing to the development of exacerbations $[6,7]$.

It is noted that monitoring of etiologically significant strains of microorganisms in patients with acute and chronic bronchopulmonary pathology is necessary to improve the pathogenetic tactics of treatment and prevention of the formation of chronic diseases $[1,7]$.

The aim of the study is to investigate the respiratory microbiota in children with severe neurological pathology in order to optimize an antibacterial therapy of recurrent respiratory diseases.

\section{Materials and methods}

The study was carried out on the basis of the infectious diseases department of the Children's Clinical Hospital No. 6 in Dnipro in 2017-2019. There were 16 children from the nursing home for the disabled aged 4 to 12 years with severe neurological pathology under our supervision. Nosological forms were presented as follows: 8 children with cerebral palsy, persistent movement disorders, symptomatic epilepsy and 8 children with congenital malformations of the brain (microcephaly, hydroanencephaly or hypoplasia of the corpus callosum and frontal-temporal parts), spastic tetraparesis, symptomatic epilepsy, severe underdevelopment of higher cortical functions. Children with disabilities were in the department for the treatment of respiratory diseases, which were of a protracted and often recurrent nature.

The study was carried out in accordance with the Declaration of Helsinki, the Council of Europe Convention about human rights, biomedicine and relevant laws of Ukraine and approved by the Bioethics Commission of Children's Clinical Hospital No. 6 (the protocol No. 4 dated 22.10.2019). The informed consent to participate in the study was received from all patients' parents.

Comprehensive examination of the patients included a detailed anamnestic analysis, physical examination of children, chest x-ray, blood test (CBC+DIFF) and biochemical analysis (protein fractions and $\mathrm{C}$-reactive protein test). The microbiological research of the upper respiratory tract using a deep smear from the oropharynx was done. The biomaterial was taken after mouth cavity washing, into a disposable sterile sealed container. Clinical samples were delivered to the laboratory within an hour after the sampling of the material and were inoculated on prefabricated nutrient substratum. Determination of a bacterial culture was carried out using the light and phase contrast microscopy. Antibiotic sensitivity was determined by the Kirby-Bauer test.

Statistica 13.0 software (serial number AGAR909E415822FA) was used for statistical processing of the obtained results. Quantitative and qualitative indicators were evaluated. The arithmetic mean value (M) and its standard error (m) were determined with a normal distribution of parameter values.

\section{Research results}

The anamnestic data analysis revealed the presence of recurrent and chronic respiratory pathology in neurological disabled children. The 7 patients $(43.7 \%)$ had repeated bronchopneumonia in the anamnesis. Three patients $(18.7 \%)$ were previously diagnosed with chronic bronchitis, which was substantiated not only by clinical and anamnestic data, but also confirmed by a bronchoscopic examination. The 6 children $(37.5 \%)$ had the repeated bronchitis with an average frequency of $5.9 \pm$ \pm 0.4 per year, which made it possible to speak of the recurrent nature of acute bronchitis. The clinical feature of the bronchitis course in children with severe neurological pathology was their protracted character. The average duration of the disease was $7 \pm 0.4$ weeks.

The bronchitis proceeded with obstructive syndrome in 6 patients $(37.5 \%)$, which was characterized by long-term preservation of characteristic auscultatory data, expiratory dyspnea. Respiratory diseases were accompanied by signs of respiratory failure of the II-III degree, there was a decrease of oxygen saturation to $93-88 \%$.

The recurrence of bronchitis and their protracted nature, without clinical symptoms of reinfection, may have been due to the presence of dysphagia syndrome and chronic microaspiration in children with severe neurological pathology. The signs of varying degrees of dysphagia were detected in all examined children on physical examination and based on the study of anamnestic data. The regurgitation was observed during feeding in $68.7 \%(n=11)$ of patients. The food was thrown into the nasal cavity in $56.3 \%(\mathrm{n}=9)$ of children. Chronic microaspiration syndrome promotes the development of an inflammatory process in the bronchial mucosa and the penetration of bacterial flora into the lower respiratory tract, which determines the features of the course of bronchopulmonary pathology in neurological patients $[8,9]$.

Based on the clinical assessment of the hematological and biochemical results, all patients were found to have leukocytosis, neutrophilia, increased ESR and Creactive protein above reference values.

The clinical and laboratory changes, which remained in dynamics, required long-term antibiotic therapy.

When studying the anamnestic data, it was found that the average duration of antibiotic therapy in children of this group was $5 \pm 0.5$ weeks, with the appointment of two and three courses in $87.5 \%(n=14)$ of cases.

In this regard, it was relevant to conduct a microbiological examination of the respiratory tract in order to identify a possible pathogen and optimize the antibacterial therapy.

The study of the microbial spectrum of the upper respiratory tract in disabled children with severe neurological pathology revealed a pronounced imbalance of the respiratory microbiota corresponding to the II and III degrees of dysbiotic disorders [7]. Gr-negative flora was detected in $43.7 \%(n=7)$ patients of the examined group with a predominance of conditionally pathogenic intestinal flora (Klebsiella pneumoniae, Proteus vulgaris, E. coli). Pseudomonas aeruginosa was identified in $37.5 \%$ $(\mathrm{n}=6)$ of cases. Also, a feature of the microbial spectrum of the upper respiratory tract in children with severe neurological pathology was the presence of a high titer of Staphylococcus aureus in $50 \%(\mathrm{n}=8)$ cases, Streptococcus pyogenus in $50 \%(\mathrm{n}=8)$ cases $)$ and yeast-like fungi of the genus Candida in $37.5 \%(\mathrm{n}=6)$ cases. The combi- 
nation of pathogenic and opportunistic flora was observed in $43.7 \%(n=7)$ of children against the background of a decrease or practically absence of normal microflora (Streptococcus spp, Neisseria spp., Aerococcus viridans).

The maximum degree of dysbiotic disorders was noted in the group of children with congenital malformations of the brain and correlated with the severity of the current broncho-pulmonary diseases.

An analysis of the sensitivity of pathogenic microflora to antibacterial agents showed high resistance of the identified microorganisms to most medicines. Pseudomonas aeruginosa had the maximum resistance, which was characterized by a weak sensitivity to antibiotics from the groups of fluoroquinolones (gatifloxacin), tetracyclines (doxycycline), cephalosporins of the 3rd and 4th generations (ceftriaxone, cefepime) and aminoglycosides (tobramycin antibiotics) and other groups of antibacterial drugs. Moderate sensitivity of Pseudomonas aeruginosa was observed only to carbapenems (meropenem).

The opportunistic intestinal flora (Klebsiella pneumoniae, Proteus vulgaris) was moderately sensitive to fluoroquinolones (levofloxacin, ciprofloxacin, gatifloxacin) and aminoglycosides (amikacin, tobramycin) in most of the examined patients. Streptococcus pyogenus and Staphylococcus aureus isolated from the mucous membranes of the oropharynx in the examined patients were also characterized by high resistance to most antibiotics.

Taking into account the sensitivity of the microflora of the respiratory tract the antibacterial therapy were prescribed to children with severe neurological pathology with exacerbation of chronic and recurrent bronchitis.

As a result of the respiratory diseases treatment in disabled children, a decrease in the duration of exacerbation was observed, with a more rapid normalization of clinical and laboratory data, which made it possible to reduce the duration of the course of antibiotic therapy.

\section{Discussion of research results}

The microbacteriological data are essential for understanding the causes of recurrence of respiratory diseases and the state of antimicrobial resistance in neurological patients.

Unfortunately, the insufficient number of studies of the respiratory microbiota in neurological patients complicates the comparative analysis of the obtained results. Most of the research in this area is devoted to the study of oral microflora in children with frequent respiratory diseases $[1,9]$. Thus, a high contamination of the nasopharynx with opportunistic microorganisms was revealed in the majority of frequently ill children. It should be noted that in $54.7 \%$ of children in this group a mixed infection was registered with seeding of 2 or more microorganisms. Haemophylus influenzae (37.3\%) ranked first in the children with repeated respiratory diseases, Str. pneumoniae in $34.7 \%$, followed by Staphylococc aureus - $26.7 \%$ [9].

Literature data about the microbial spectrum of the respiratory tract in children with acute and recurrent bronchitis are quite contradictory. The analysis of the comparison of the microbiocenosis of the oropharynx in children with acute and chronic bronchitis compared with the same indicator in clinically healthy children revealed normocenosis in $48.1 \%$ of cases and an intermediate type of respiratory microbiota disorder in $44.4 \%$. Oropharyngeal dysbiosis was extremely rare (in $7.4 \%$ of children). There were no significant differences in the type of microbiocenosis of the oropharynx in patients with acute and chronic bronchitis $[5,10]$.

The respiratory microflora in severe neurological patients was studied by bacterial analysis of the bronchoalveolar fluid. Thus, microbial analysis of bronchoalveolar lavage in children with severe neurodisability did not reveal Pseudomonas aeruginosa, only Streptococcus mitis was identified [11]. However, the authors noted that bacterial airway colonization by oral commensals may play a role in both chronic respiratory symptoms and inflammation.

According to our data the composition of the oropharynx microflora in patients with neurological disabilities was distinguished by a various spectrum of pathogenic microorganisms, which is not typical for this biological locus $[1,3]$.

Study limitations. The limitation of the study is the lack of routine bacteriological screening of the respiratory microflora in patients receiving antibiotic therapy in a hospital and the difficulty of the study of severe neurological patients. Thus, it is difficult to identify the entire spectrum of the respiratory microflora disordes in neurological disabled children using a limited amount of research.

Prospects for further research. In our opinion, a promising direction for further research will be a comparative analysis of the oral microflora with the respiratory microbiota of the broncho-alveolar fluid in neurological disabled children.

\section{Conclusions}

1. Respiratory pathology in patients with severe neurological disorders is characterized by recurrent and chronic bronchitis with a tendency to protracted course, often complicated by broncho-obstructive syndrome, which determines the duration of antibiotic therapy.

2. A specific feature of the microbial spectrum of the upper respiratory tract in children with repeated respiratory diseases against the background of severe neurological pathology is the dominance of Pseudomonas aeroginosa, an intestinal opportunistic microflora (Klebsiella pneumoniae, Proteus vulgaris), with a frequent combination with Streptococcus pyogenus, Staphylococcus aureus and fungi of the genus Candida.

3 . The use of a non-invasive technique for studying of the upper respiratory tract microbiota allowed to identify etiologically significant microorganisms of recurrent respiratory diseases in disabled patients.

\section{Conflict of interests}

The authors declare that they have no conflicts of interest. 


\section{References}

1. Metelskaya, V. A., Alshkin, V. A., Voropaeva, E. A., Karaulov, A. V., Nesvizhsky, Yu. V., Afanasyev, S. S. et. al. (2010). Colonization resistance and immunological reactivity of children's oropharyngeal mucosa in health and bronchopulmonary pathology. Annals of the Russian academy of medical sciences, 7, 10-15.

2. Charlson, E. S., Bittinger, K., Chen, J., Diamond, J. M., Li, H., Collman, R. G., Bushman, F. D. (2012). Assessing Bacterial Populations in the Lung by Replicate Analysis of Samples from the Upper and Lower Respiratory Tracts. PLoS ONE, 7 (9), e42786. doi: http://doi.org/10.1371/journal.pone.0042786

3. Zakharova, I. N., Kasjanova, A. N., Klimov, L. Y., Kurianinova, V. A., Simakova, M. A. et. al. (2018). Respiratory tract microbiome: what is known today? Pediatrics (Suppl Consilium Medicum), 4, 10-17. doi: http://doi.org/10.26442/24138460.2018.4.180129

4. Ilchenko, S. I., Duka, E. D., Zhukova, L. A. (2016). Microaspiration Syndrome in Pediatric Practice: Modern Features and Role in Bronchial Obstruction Syndrome Formation. Child`s health, 7 (75), 90-94. doi: http://doi.org/10.22141/22240551.7.75.2016.86731

5. Metelskaia, V. A. (2013). Kharakteristika kolonizatsionnoi rezistentnosti slizistykh obolochek dykhatelnogo trakta pri bronkhitakh u detei. Moscow, 119.

6. Lupaltsova, O. S. (2014). Osobennosti mikroflory dykhatelnykh putei u detei s respiratornoi patologiei. Sovremennye aspekty infektsionnoi patologii. Astrakhan, 94-99.

7. Afanasev, S. S., Aleshkin, V. A., Voropaeva, E. A., Afanasev, M. S., Slobodeniuk, V. V., Karaulov, A. V. (2013). Microbiocenoses of open cavities and mucosal immunity. Effektivnaia farmakoterapiia, 1 (27), 6-11. Available at: https://umedp.ru/upload/iblock/87d/2.pdf

8. Samatova, E. V. (2012). Microbiocenosis Characteristics of the Low Respiratory Tract at Chronic Infectious-Inflammatory Pulmonary Diseases in Children and Antibiotic Resistance of Basic Pathogens. Journal of Ural Medical Academic Science, 1 (38). Available from: http://vestnikural.ru/article/osobennosti-mikrobiocenoza-nijnih-dyhatelnyh-putey-pri-hronicheskih-infekcionnovospalitelnyh-zabolevaniyah-legkih-detey-i-antibiotikorezistentnost-osnovnyh-patogenov

9. Kamasheva, G. T., Belukhina, E. G., Sharipova, G. K., Karipollin, B. K. (2011). Kharakteristika mikrobiotsenoza verkhnikh dykhatelnykh putei u chasto boleiuschikh detei g. Semei. Nauka i zdravookhranenie, 1, 69-71.

10. Huffnagle, G. B., Dickson, R. P. (2015). The bacterial microbiota in inflammatory lung diseases. Clinical Immunology, 159 (2), 177-182. doi: http://doi.org/10.1016/j.clim.2015.05.022

11. Trinick, R. E., Bunni, L., Thorburn, K., Hackett, A. P., Dalzell, M., McNamara, P. S. (2015). An Observational Study Examining the Relationship between Respiratory Symptoms, Airway Inflammation and Bacteriology in Children with Severe Neurodisability. PLOS ONE, 10 (4), e0124627. doi: http://doi.org/10.1371/journal.pone.0124627

Received date 03.12.2020

Accepted date 28.12.2020

Published date 30.01.2021

Olena Koreniuk, PhD, Associate Professor, Department of Propaedeutics of Children's Diseases, State Institution "Dnipropetrovsk Medical Academy of the Ministry of Health of Ukraine" V. Vernadskogo str., 9, Dnipro, Ukraine, 49044

E-mail: okoreniuk@ukr.net 